\title{
A New Design of Multi-Channel Inductance to Digital Converter Based on LDC1612
}

\author{
Huang Jian \\ XiJing University, Xi'an 710123, China; \\ 565200245@qq.com
}

Keywords: LDC1612; IIC interface; MSP430F5529

Abstract. Inductive sensing is a contactless, magnet-free sensing technology that can precisely measure the position, motion, or composition of a metal or conductive target as well as detect the compression, extension, or twist of a spring. Nowaday inductive measure method have limited in high power dissipation、 lower accurate etc. In order to resolve this problem, A detection device was introduced, which based on 2-channels inductance-to-digital converters LDC1612, used PCB coils as inductive sensor to detect metal, converted the sensor signals into digital signals, used an IIC interface connection to an untra-low power MSP430F5529 in order to implement 2-channels high-speed data transmission. The test results show that Sub-micron resolution in position-sensing applications with up to 28-bits of resolution, the device can be used in harsh environments, offered contactless sensing that is immune to nonconductive contaminants such as oil, water, dirt and dust, implemented 2-channels contactless inductive sensor which has better performance, better reliability, and greater flexibility than existing sensing solutions - at lower overall system cost and power.

\section{Introduction}

Induction sensing is a non-contact, no magnet induction technology, using the coil inductance and mutual inductance changes to achieve non electrical measurement, the use of electromagnetic induction law will be measured non electric energy into electricity. Such sensors are mainly used for displacement measurement and measurement of mechanical quantities that can be converted into displacement changes. Compared with other types of sensors, this sensor provides non-contact sensing, from non conducting pollutants (e.g., oil, water, dirt and dust) interference, sensor placed away from the position of the IC - very suitable for use in harsh environments, detection is widely used in scientific research, industrial production. But the frequency response of common induction sensors is very low, so it is necessary to design external signal conversion circuit to output power. The equipment is complex, large size, high power consumption and high cost.

In this paper, using the most advanced 2 channel 28 bit high precision inductance digital converter LDC1612 as the sensor probe, the chip with digital IIC interface, can be easily connected to various low power MCU for data acquisition and processing. LDC1612 in non-contact sensing, induction by winding coil, PCB coil or spring, with internal signal conversion circuit, the induction signal is converted to 28 digit volume, the position and movement of components or precise measurement of metal or conductive targets.

\section{System Design}

System design, the main control using MSP430F5529, the MCU is TI formula produced by a low-power microprocessor, powerful. The IIC interface is connected with LDC1612, object PCB inductance coil to detect metal LDC1612 front end, different distance will return the number of different amount, calculated by MCU digital quantity received between the metal and the coil distance, and then sent to the display unit for a liquid crystal display. 


\section{Detailed design and key technologies}

The hardware connection between the MSP430F5529 and the LDC1612 is shown in figure 1. High speed data transmission is realized by interconnecting two data lines of SDA and SCLK. MSP430F5529 reads and writes data to LDC1612 as shown in Figure 3 and figure 4. Communication follows standard IIC communication protocol. When reading and writing, the master and slave address is sent first, and then the data.

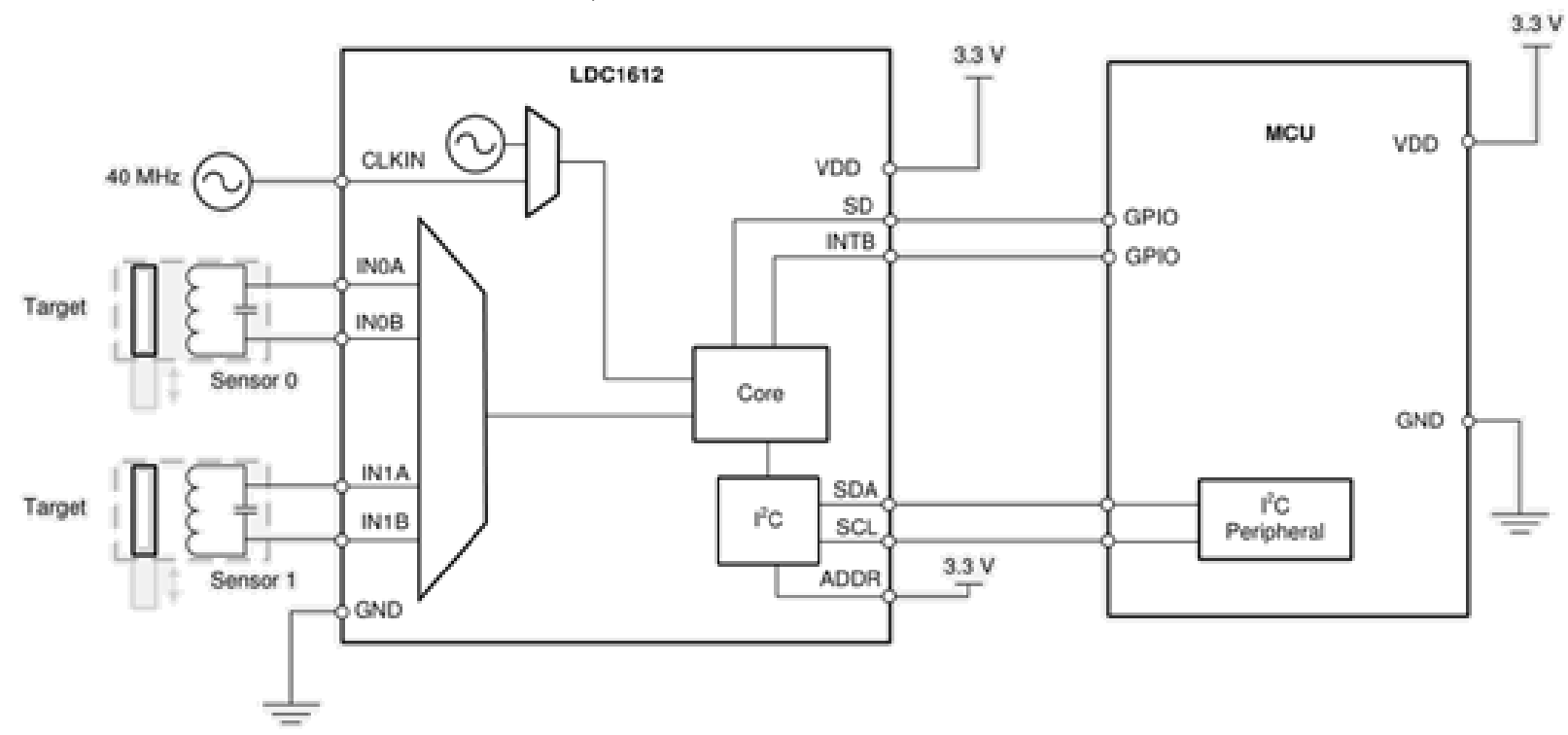

Figure 1 hardware connections between MSP430F5529 and LDC1612

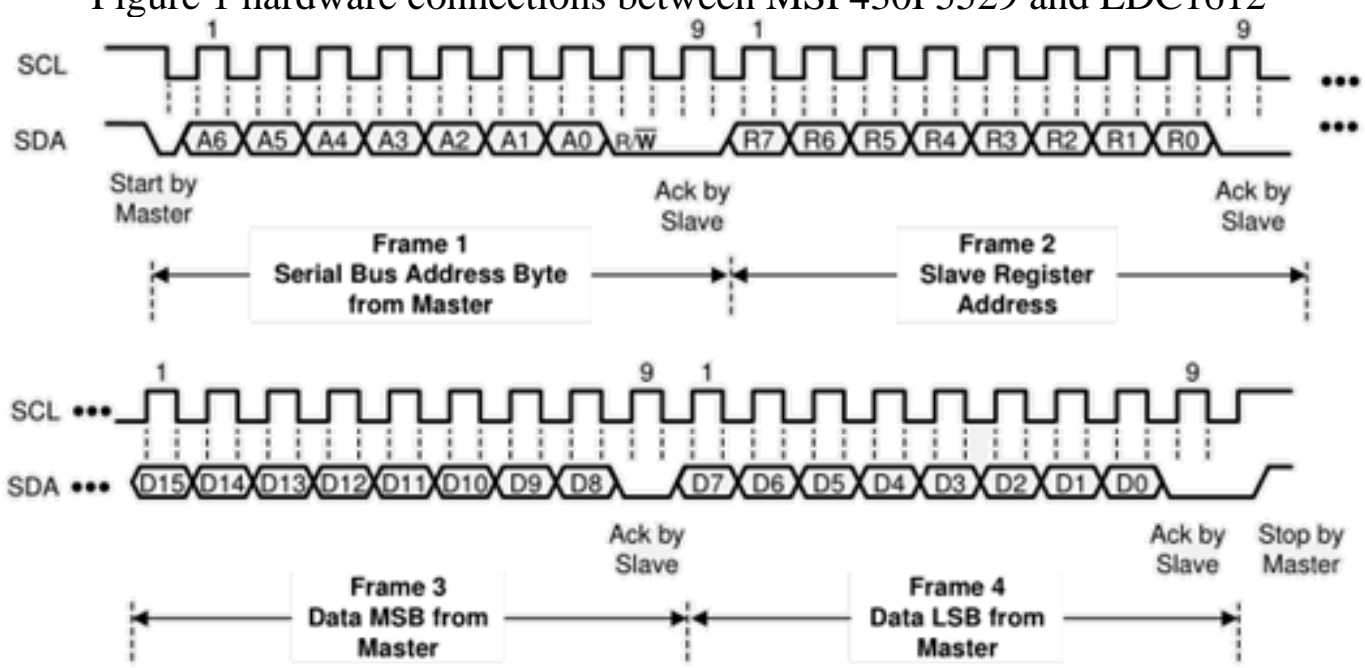

Figure 2 IIC write register timing
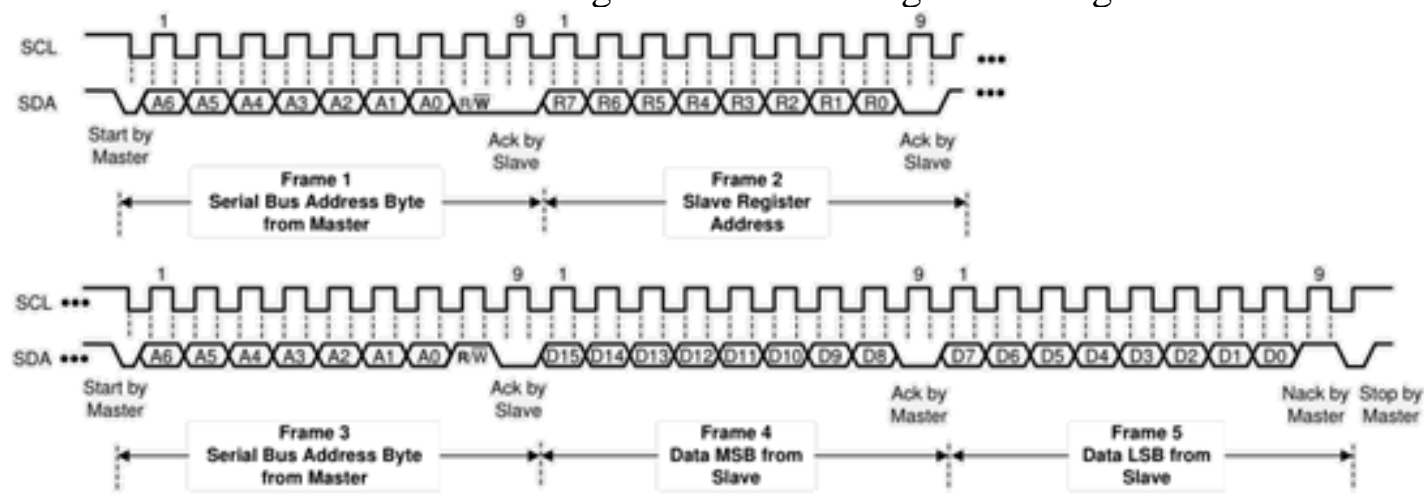

Figure 3 IIC read timing 


\section{Software Program}

In this design, under KEIL 5, programming with C language. Achieve the initialization of LDC1612 and read and write operations. Some code is given below:

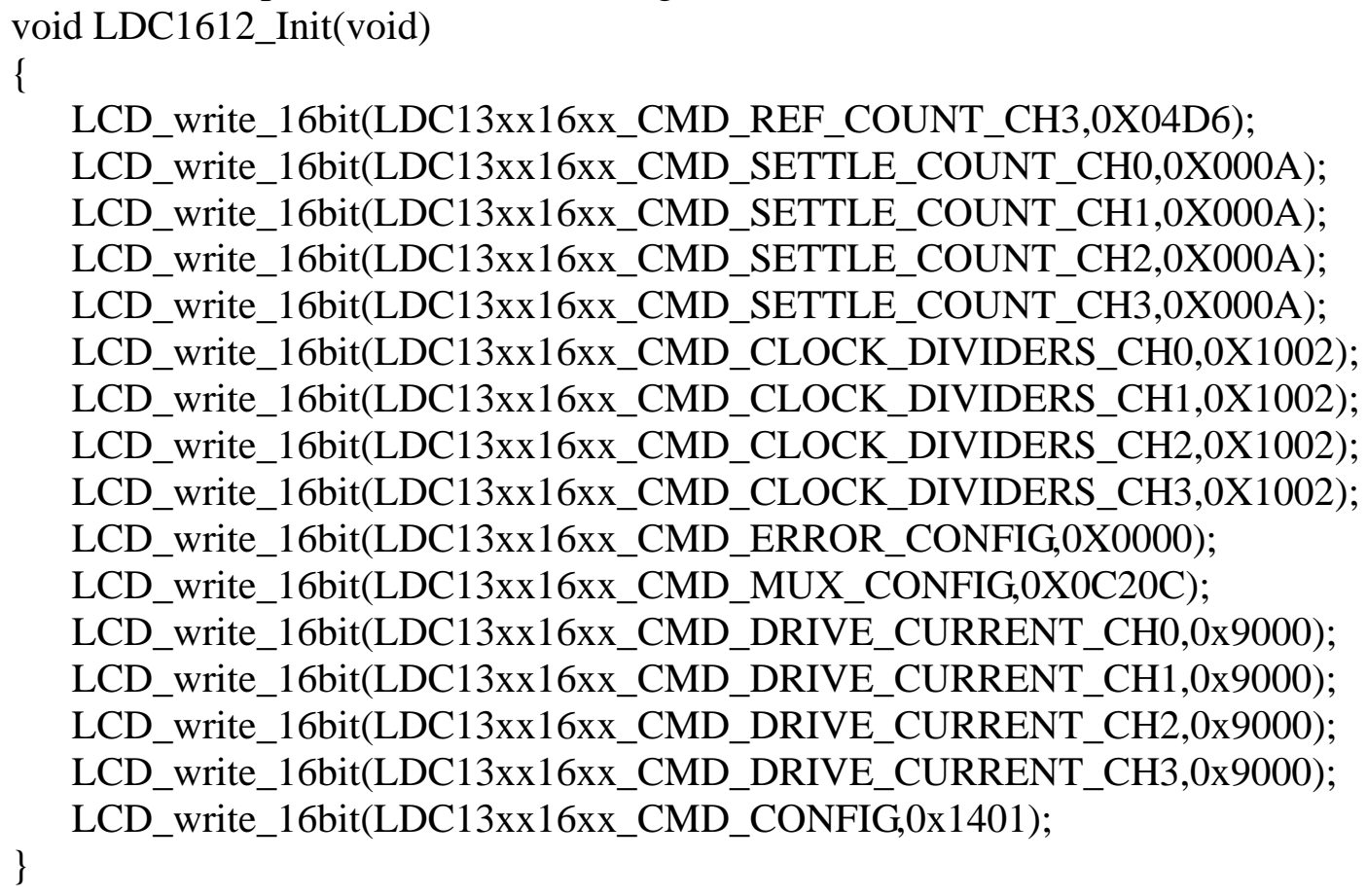

\section{Conclusion}

This paper describes the design of a multi channel digital converter based on LDC1612 inductance, the ultra low power MSP430F5529 as a master, with LDC1612 as the detecting device, a careful analysis of the working principle of LDC1612 and related parameters, realize high speed data acquisition through the IIC interface, to reduce power consumption, reduce the volume, digital interface, digital high up to 28 , can realize non-contact metal detection, distance from $0 \mathrm{~mm}$ to $100 \mathrm{~mm}$, the precision is close to UM level. Because of its strong anti-interference ability, it can be used in dust, dirt, oil and humidity and other harsh environment. Its multi-channel sensing capability supports foil or conductive ink targets, providing unlimited possibilities for creative and innovative system design.

\section{Reference}

[1]Texas Instruments.LDC1612 Multi-Channel 28-Bit Inductance to Digital Converter (LDC) for Inductive Sensing[M].Shanghai, 2014，12。

[2]Texas Instruments.LDC Sensor Design[M].Shanghai, 2015,3

[3] Guo Yu, Li Yanmei, Wang Peng. Design of coin discrimination system of eddy current sensor based on [J] 2012,25 Journal of transducer technology, (4): 557-560

[4]TI.LDC1612, Evaluation, Module, User, s, Guide[M].AMERICAN,, 2014, 17-18.

[5] Shi Yanping, Liu Chengwen, Ni Lixue. Dynamic track of amorphous alloy pressing magnetic research on the inductive stress sensor based on Journal of sensor technology [J].2009,22 (12): 1704-1708. 